\title{
Diacronie
}

Studi di Storia Contemporanea

$\mathrm{N}^{\circ} 27,3$ | 2016

Stato, costituzione e democrazia

\section{Epistemologia della democrazia e modernità. La posta in gioco del superamento dell'Illuminismo in Europa occidentale}

\section{Georgios Contogeorgis}

Traduttore: Irene Vagionakis

\section{(2) OpenEdition Journals}

\section{Edizione digitale}

URL: http://journals.openedition.org/diacronie/4321

DOI: $10.4000 /$ diacronie.4321

ISSN: 2038-0925

\section{Editore}

Association culturelle Diacronie

\section{Notizia bibliografica digitale}




\title{
Diacronie
}

\section{Epistemologia della democrazia e modernità. La posta in gioco del superamento dell'Illuminismo in Europa occidentale *}

\author{
Georgios CONTOGEORGIS *
}

traduzione a cura di Irene VAGIONAKIS

Quella che siamo abituati a chiamare democrazia è davvero sempre democrazia? Rispondendo negativamente a questo ossimorico quesito, l'autore si propone di esaminare tale ambiguo concetto dal punto di vista dell'epistemologia cosmosistemica. Mettendo in luce la superficialità epistemologica della modernità, rea di non saper cogliere la natura delle forme costituzionali oggi vigenti in Europa occidentale, l'articolo va a dimostrare come i governi attuali non siano democrazie, né tanto meno costituiscano il punto di arrivo dell'evoluzione istituzionale del cosmosistema antropocentrico, in quanto ne incarnano piuttosto una fase prerappresentativa proto-antropocentrica. L'articolata argomentazione si svolge attraverso la delineazione del fine della democrazia (la concretizzazione della libertà individuale, sociale e politica), la descrizione del suo sistema socio-economico e politico, l'analisi contrastiva fra questa ed i governi non democratici ed infine la contestualizzazione della democrazia nel suo milieu storico, mediante una panoramica sull'evoluzione diacronica delle forme di governo proprie del cosmosistema antropocentrico.

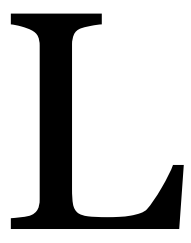

a transizione dell'Europa occidentale dal cosmosistema despotico (il feudalesimo) a quello antropocentrico, in combinazione con il passaggio di quest'ultimodalla piccola alla grande scala cosmosistemica (dalla cittàStato allo Stato-nazione), ha portato prepotentemente in primo piano la 
questione della democrazia. Allo stesso tempo, però, la democrazia come concetto ha assunto un'ambiguità senza precedenti, come risultato del suo debito ideologico e della carenza epistemologica della scienza moderna.

Nelle pagine che seguono tenterò di decifrare quest'avventura della democrazia e di avvicinarmi al concetto dal punto di vista dell'epistemologia cosmosistemica. Per prima cosa determinerò la problematica relazione fra la modernità e l'epistemologia della democrazia. Successivamente mostrerò il suo fine. Come terza cosa delineerò il suo sistema, che è inteso a realizzare lo scopo della democrazia. Come quarta cosa descriverò il contrasto fra la democrazia e le altre forme di governo pertinenti al cosmosistema antropocentrico. Da ultimo indicherò il tempo e il luogo (il quando e il dove) della democrazia nella "biologia" antropocentrica.

\section{La superficialità epistemologica e la natura proto-antropocentrica della modernità}

La domanda fondamentale con la quale, a mio avviso, la nostra epoca si trova a confrontarsi è questa: in che modo si strutturerà un sistema di conoscenza che non funzioni soltanto in modo delucidativo, registrando i fenomeni sotto osservazione o addirittura anteponendo il presente come valore assoluto e le sue realtà come misura, cioè come standard per la comprensione o l'interpretazione del passato, e che non rimanga inoltre prigioniero della convinzione che il futuro sarà legato al corrente ordine cosmosistemico?

L'epistemologia deve proporre un sistema di conoscenza globale che attinga la propria sostanza dalla totalità della cosmostoria e che traduca i fenomeni con concetti di classe, la loro tipologia e il loro divenire evolutivo impiegando come criterio la biologia cosmosistemica dell'uomo sociale. L'epistemologia cosmosistemica ambisce a rispondere a tale sfida ${ }^{1}$.

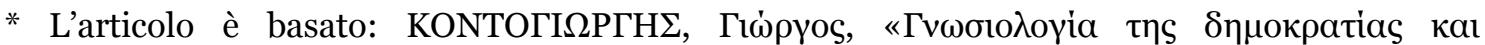

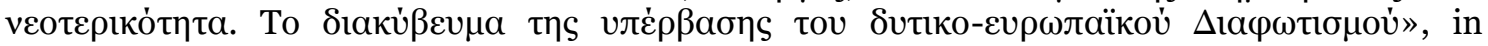
$\Delta i \alpha ́ \lambda o \gamma o s, 4$, 2014, pp. 229-247. Rispetto al testo originario l'autore ha apportato alcune modifiche, che compaiono nella traduzione italiana del saggio.

${ }^{1}$ L'epistemologia cosmosistemica implica la distinzione fra cosmostoria e storia cosmosistemica, al fine di avvicinarsi al fenomeno sociale secondo la classe della sua formazione, cioè della sua individualità, e della sua semiologia evolutiva. Il concetto di cosmosistema definisce un insieme di società con parametri di base comuni, determinanti istituzionali, ideologici e valoriali che compongono un intero, il quale si muove nel tempo della storia secondo i termini dell'autarchia interna e della coerenza evolutiva. Distinguiamo l'esistenza di due modelli cosmosistemici, quello despotico e quello antropocentrico. Quello despotico genera società di "sottomessi" alla proprietà di terzi, quello antropocentrico società aventi come segno distintivo la libertà. Il cosmosistema antropocentrico è distinto in due macro-periodi: quello di piccola scala, incarnato 
In tale cornice, mi focalizzerò nello specifico sulla questione epistemologica che riguarda il concetto di democrazia per verificare, ad un primo livello, se il progetto costituzionale e, di conseguenza, il sistema politico che la modernità ha elaborato dal Rinascimento e dall'Illuminismo nell'Europa occidentale e che è fondamentalmente accettato ai nostri giorni, sia parte della democrazia. Se conveniamo che questo sistema si classifichi davvero fra i governi democratici, come riconoscono essenzialmente tutti gli intellettuali della scienza contemporanea e la classe dirigente, significa che non occorre continuare il dibattito ma piuttosto fare come fa l'attuale istruzione, ovvero prendere come dato acquisito il fatto che il sistema odierno sia una democrazia e continuare a descriverne le istituzioni: il parlamento, il governo, i partiti, le lobby, il diritto di voto e di parola e le varie altre istituzioni, valori e prassi che fanno parte della nostra quotidianità.

Ciononostante, è evidente che questa scelta sia scientificamente un vicolo cieco, poiché aggira l'obiettivo, l'epistemologia del fenomeno, al fine di classificare per partito preso come democrazia la forma di governo che la modernità vive, ritenendola per di più superiore alla democrazia della città-Stato che ha generato il concetto. Un'ulteriore conseguenza di questa scelta è l'affermazione che una qualsiasi opposizione all'odierna realtà costituzionale sia imputabile di collaborazionismo con l'autocrazia, con il sistema politico autocratico. O si sceglie l'una o si sceglie l'altro.

Questo approccio non tiene conto evidentemente del fatto che la nostra epoca appartiene tipologicamente a una fase che si fonda sui principi dell'unità di pensiero e azione e dell'esistenza di una e una sola proposta di governo, indipendentemente dalle sue differenziazioni morfologiche. Mi riferisco al periodo proto-antropocentrico del nuovo mondo, che è emerso alla sua uscita dal feudalesimo/despotismo. Nonostante ciò, la modernità lascia trasparire come il suo modello sia unico ed essenzialmente definitivo, assieme al concetto della realizzazione antropocentrica, e che la prospettiva dello sviluppo, della mutazione del presente, dunque, non si muova verso un governo tipologicamente differente. È pronta ad accogliere la possibilità di alcuni adattamenti morfologici, i quali non cambieranno però l'essenza dell'attuale democrazia.

Questa stasi è, a mio parere, ideologicamente conservatrice ed epistemologicamente insostenibile, in quanto nega la natura evolutiva dell'uomo sociale e, nello specifico,

essenzialmente dall'ellenismo, e quello di grande scala, rappresentato dall'età moderna. Il cosmosistema antropocentrico di piccola scala può mostrare un percorso evolutivo completo, elemento fondamentale per lo studio del fenomeno sociale, mentre l'epoca della grande scala cosmosistemica si presenta solamente come proto-antropocentrica. Approfondimenti su questo

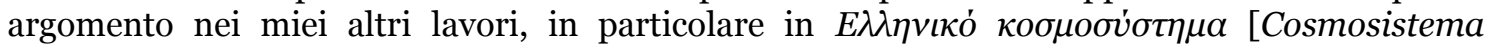
ellenico], Atene, Edizioni Sideri, 2006. 
della società antropocentrica. Questo approccio del divenire evolutivo dell'uomo sociale si combina, però, con il fatto che la modernità non sia riuscita finora a porre una qualche problematica credibile per lo sviluppo che possa andare oltre ai fondamenti costituzionali del presente. Ciò diventa chiaro quando ci accostiamo ai concetti che riguardano l'uomo sociale e, nella fattispecie, a quelli che sono compagni del concetto di democrazia, come la libertà, l'uguaglianza, la giustizia, il diritto, la cittadinanza, e via dicendo. Tutti questi concetti, proprio come il concetto di democrazia, hanno necessariamente una sostanza specifica ed essenzialmente descrivono le realtà che vive il mondo oggi, le quali emergono come posta in gioco epistemologica globale.

Per comprendere la causa di questo fenomeno occorre tornare all'epoca dell'ingresso del mondo feudale dell'Europa occidentale nell'età moderna. A quei tempi, il contatto con il concetto di democrazia non era frutto delle circostanze, cioè della realtà, sì che gli intellettuali potessero attingere da questa la sua sostanza ed essere quindi condotti ad una definizione che descrivesse il loro tipo di governo. Il mondo moderno ha scoperto la democrazia e si è informato sulla sua esistenza, nel percorso verso la transizione antropocentrica, attraverso la letteratura greca.

Proprio per questo è avvenuto che gli intellettuali non siano riusciti a capire la posta in gioco e a comprendere la sostanza dell'armamentario istituzionale della democrazia, e che si siano trovati in una posizione tale da non riuscire a tenere conto del tempo in cui è fiorita. Assieme però alla mancanza del sostegno che i presupposti intellettuali avrebbero potuto fornire per la comprensione del concetto di democrazia, nemmeno le circostanze sono state d'aiuto per accettarla come azzardo fattuale e a svilupparla. Certamente le priorità dell'epoca della transizione dal cosmosistema despotico a quello antropocentrico hanno sollevato in modo univoco la questione della libertà individuale, cioè della rimozione dei vincoli della proprietà feudale sull'individuo. Questa libertà non contrasta comunque con il fatto che il governo sia proprietà di terzi - come nello Stato assolutistico o nello Stato-nazione -, né apparentemente con il fatto che il sistema dell'economia sia parte della proprietà (dell'imprenditore, del capitalista o anche dello Stato) diversificata dalla società o dai suoi singoli elementi.

Il caso degli Illuministi è estremamente emblematico di come, nella loro incapacità di avvicinarsi all'essenza e cioè all'obiettivo, alle istituzioni e al tempo della democrazia - così come dei concetti ad essa connessi, come la libertà -, siano giunti alla costruzione dell'ideologia della modernità. Questa consiste nella "dimostrazione" della classificazione democratica della forma di governo che gli Illuministi hanno delineato per il periodo successivo alla liberazione dal despotismo. In tale contesto, era naturale che l'uomo intellettuale dell'epoca della transizione si allontanasse dall'ammirazione 
iniziale per l'“Antichità”, e dunque per la democrazia, per rispondere alle domande urgenti che si trovava ad affrontare: come si sarebbe strutturata la nuova società antropocentrica, per lui senza precedenti, avente come solo segno distintivo la libertà individuale; come il sistema socio-economico e politico avrebbe trovato un equilibrio nel contesto di una società antropocentrica in considerazione del fatto che questo faceva parte della proprietà; come si sarebbe legittimato infine il rifiuto della democrazia della città - della città-Stato ma anche della città/confederazione dell'epoca -, senza che venisse mossa all'emergente assetto politico l'accusa di oligarchia; quale sarebbe stato il suo obiettivo, insieme a molte altre questioni.

Attraverso complessi processi cognitivi ed invenzioni istituzionali, che dominano ancora ai nostri giorni, si è raggiunta infine l'inclusione fra le democrazie di qualsiasi necessario sistema costituzionale (economico, sociale e politico) della prima epoca post-feudale; tali sistemi però, dal punto di vista dell'epistemologia cosmosistemica, corrispondono ad esempio a governi rigidamente oligarchici aventi come segno la monarchia elettiva: si classificano cioè come oligarchie governate da un solo individuo, il cui corpo, non ereditario, è contraddistinto da un termine temporale definito.

Ciò che comunque suscita interesse nella nostra epoca è che il dibattito sulla democrazia e sui concetti relativi alla sua sostanza continua ad avvenire entro i termini dell'Illuminismo, mentre è verificabile persino ad occhio nudo che quanto avviene davanti a noi, ai nostri giorni, indica che stiamo vivendo non una crisi interna al periodo proto-antropocentrico, bensì un cambiamento di fase, il quale rende necessario un cambiamento del modello valoriale e sistemico.

\section{L'obiettivo della democrazia}

La democrazia non è fine a se stessa. È il governo che è chiamato a realizzare l'obiettivo della società antropocentrica, cioè la libertà. Nella fattispecie, la libertà della democrazia copre la totalità della vita sociale degli uomini. Per schematizzare, potremmo dire che la democrazia è chiamata ad istituzionalizzare il dominio sociale così che la libertà dei suoi membri si concretizzi nei campi della vita individuale/privata, di quella socio-economica e di quella politica.

Questa ammissione rende necessaria la chiarificazione di una serie di questioni. Prima di tutto, il concetto di libertà si definisce come autonomia, include cioè nella propria sostanza il presupposto autodecisionale dell'individuo in ciò che riguarda tanto la vita personale o privata quanto la sfera socio-politica. Il principio dell'autonomia nella sua formulazione democratica risale alla massima del «non essere governato da 
nessuno». La libertà individuale introduce il presupposto dell'autonomia nella vita privata di ciascuno nella società. La libertà sociale interessa i campi della vita sociale nei quali l'individuo stipula contratti, come ad esempio i (sotto-)sistemi economici. La libertà politica riguarda il posto dell'individuo membro della società nel suo complesso, cioè la relazione tra società e politica.

Di conseguenza, la libertà totale (individuale, sociale, politica) indica che il governo come intero, ovvero il sistema economico, sociale e politico, deve salvaguardare l'autonomia e cioè l'indipendenza dell'individuo da terzi in ogni campo. Affinché ciò avvenga occorre che l'individuo o faccia parimenti parte dell'amministrazione del sistema o non sottostia alla sua dipendenza. La scelta dell'una o dell'altra soluzione è legata essenzialmente al tempo antropocentrico ${ }^{2}$. Questo significa che nel campo dell'economia, ad esempio, il sistema si preoccupa di non inserire il cittadino nella semiologia della dipendenza del settore del lavoro o, diversamente, di definirlo come associato interno al sistema. Nel campo della politica la libertà presuppone l'incarnazione della totalità del sistema da parte del corpo della società dei cittadini, anziché dello Stato.

Questa considerazione solleva la questione del posto della proprietà nella democrazia. Certo, la democrazia non si oppone alla proprietà. Al contrario. Distingue però tra la proprietà individuale, laddove questa non implichi la stipulazione di contratti con terzi, nello specifico con il cittadino, e la proprietà che potrebbe cancellare la libertà. I più tipici fra questi contratti interessano i campi del sistema economico e politico.

Un altro parametro, del tutto ideologico, che la modernità presenta per dimostrare l'esercizio della libertà totale da parte della democrazia riguarda il concetto di consenso. Il consenso è stato chiaramente addotto come argomento per dissimulare il fatto che la permanenza fra i suoi sistemi, nel contesto dell'antropocentrismo individuale, del precedente regime ordinato secondo rapporti di possesso, ostacolava la libertà (sociale e politica). Malgrado ciò, viene ignorato che il consenso, nel caso specifico, patrocina la legittimazione ma non la libertà. Chi acconsente non rimane libero solamente in quanto accetta la propria soggezione a un regime di dipendenza, cioè di eteronomia.

Al fine di correggere del tutto questa affermazione, la libertà in campo sociale e politico andrà definita impiegando come criterio il concetto di diritto anziché quello di

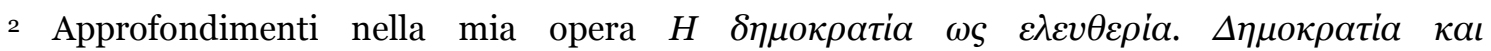

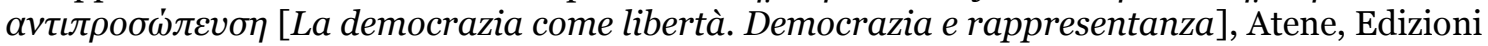
Pataki, 2007.
} 
autonomia. Questo non entra in contraddizione solo con l'approccio della libertà individuale, che avviene dal punto di vista dell'autonomia, ma pone anche il diritto come superiore alla libertà, nel momento in cui questo delimita solo estensivamente il campo della libertà individuale o il contesto della sua salvaguardia laddove l'individuo venga privato della libertà. L'odierno spazio lavorativo o la relazione tra società e politica ne costituiscono esempi emblematici. Di conseguenza la modernità, definendo la libertà sociale e politica nei termini del diritto, evita di trattare della loro assenza nei rispettivi campi, al fine di includere il proprio sistema fra i governi democratici.

Conclusione inevitabile di questa palinodia ideologica è l'affermazione della scienza moderna secondo cui la libertà individuale sia incompatibile con la libertà politica (l'equivalente uguaglianza e via dicendo), identificata però con il presunto settore della collettività sociale, cioè lo Stato/sistema e non con la società dei cittadini. $\mathrm{Al}$ contrario, l'epistemologia cosmosistemica indica che la libertà è unica, mentre i campi della sua realizzazione si aggiungono in modo cumulativo e, nello specifico, secondo il seguente ordine: libertà individuale, sociale e politica. In altre parole, non è concepibile che qualcuno persegua prima la libertà politica e poi quella individuale o che sia politicamente libero senza essere individualmente padrone di sé. La biologia delle società ha una logica fattuale ed evolutiva comune, a questo proposito, proprio come la biologia di ciascun uomo. La differenza sta nel fatto che la biologia evolutiva di un ciascun individuo è facilmente comprensibile, mentre la biologia sociale presuppone altri processi di assunzione, che la scienza moderna è lontana dal decifrare.

L’incapacità della scienza moderna nell'accostarsi alla democrazia e, per estensione, al suo obiettivo, l'ha condotta a proclamare il suo carattere totalitario. Questa asserzione non considera, malgrado ciò, il fatto che il totalitarismo, come anche l'assolutismo e l'autoritarismo, rivendicano l'esistenza di una chiara differenziazione tra il detentore della responsabilità politica totale e il suo soggetto sociale: rivendicano cioè la trasformazione della prima in una autorità politicamente dominante. Nella democrazia manca il soggetto della dominazione politica - la società dei cittadini poiché la libertà politica elimina la relazione dominante-dominato, dal momento che il sistema politico va a fare interamente parte della società dei cittadini.

In conclusione, l'obiettivo della democrazia riguarda la libertà totale - cioè in modo cumulativo libertà individuale, sociale e politica -, la quale si definisce dal punto di vista dell'autonomia dei membri del corpo sociale in campo privato, socio-economico e politico. 


\section{Il governo, ovvero il sistema socio-economico e politico della democrazia}

L'obiettivo della libertà totale può essere conseguito solo mediante il governo democratico. Questa forma di governo è chiamata ad attuare la libertà oltre al campo individuale, in quelli del sistema socio-economico e politico. La sostanziazione individuale dell'uomo può essere conservata anche in un ambiente costituzionale che accetta l'appartenenza del sistema alla proprietà (diversificata) e in ciò si allontana dalla società dei cittadini, a patto che il possessore del sistema consideri tra le sue politiche l'acquisito della libertà individuale, cioè le previdenze istituzionali, valoriali e via dicendo che la salvaguardano, e una redistribuzione della ricchezza in grado di sostenerla.

In realtà, però, questo azzardo si compie a condizione del mantenimento di un delicato equilibrio tra la società dei cittadini e i settori del sistema, in quanto le politiche degli ultimi passano attraverso relazioni di forza che si sviluppano nel contesto della dinamica sociale. La società rimane privata e trova punti di incontro o di dialogo con i settori del sistema al di fuori di questo, su una base extra-istituzionale.

Per diventare possibile la libertà sociale deve ideare un sistema che non porrà nella dipendenza di potere (contrattuale o meno) l'individuo membro della società. Come avverrà ciò? Per quanto riguarda ad esempio l'economia possiamo ipotizzare due possibilità: la prima, che l'individuo si disgiunga dal processo di produzione o acquisisca un proprio livello padronale. La seconda, che entri nel sistema come suo elemento. Non è necessario occuparsi più approfonditamente di tale questione, in quanto rimanda a un dibattito per il quale la scienza moderna non è preparata. $\mathrm{E}$ sufficiente richiamare qui il modello antropocentrico ellenico della città-Stato esclusivamente riguardo a un preconcetto: nel periodo cratocentrico della democrazia la soluzione della questione della libertà sociale è avvenuta mediante il rifiuto da parte del cittadino del processo economico (è il caso della società del tempo libero). Nella fase ecumenica del cosmosistema ellenico/antropocentrico, la libertà sociale è stata conseguita mediante l'organizzazione associativa del sistema economico3.

La libertà politica, da parte sua, può essere conseguita in un modo soltanto: con l'incarnazione del sistema politico da parte del corpo della società dei cittadini. Affinché ciò avvenga sono necessarie due cose. La formazione della società come popolo, cioè

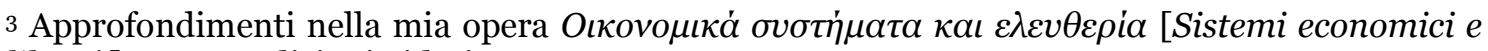
libertà], Atene, Edizioni Sideri, 2010. 
come componente organica del sistema politico, e allo stesso tempo il passaggio a questa della responsabilità politica totale, poiché solo con l'incarnazione totale del governo da parte della società l'individuo/cittadino si libera dalla tutela politica e, in ogni caso, da qualsiasi "contratto" di dipendenza.

In questo modo avviene la transizione dallo Stato dell'autorità politicamente dominante alla responsabilità politica totale ordinata in modo non autoritario della società-popolo, dalla collettività sociale che incarna e amministra lo Stato (i suoi possessori, e cioè la nazione dello Stato) alla collettività sociale che si definisce come la controparte tautologica della società-popolo (la nazione della società).

Il rifiuto della divisione delle competenze politiche - e chiaramente, per quanto riguarda la libertà sociale, di quelle sociali - costituisce il presupposto per l'abolizione dell'eteronomia di potere. Cosa che indica che l'invocazione e nello specifico l'applicazione di questa autorità non sottostà alla causalità dell'autorità della cosiddetta complessità, richiamata dall'ideologia oligarchica, dipende invece dal grado della realizzazione antropocentrica dell'uomo sociale. La totalità dell'ambiente istituzionale della democrazia va inalterata a fare parte della società-popolo. Ogni autorità esecutiva è chiamata soltanto ad eseguire restrittivamente, come fosse un servitore, le politiche del popolo.

Si fa infine chiaro che la massima della democrazia - «il non essere governato da nessuno» - non assimila questa forma di governo all'anarchia, poiché la seconda non costituisce una forma di governo. D'altra parte, la sua stessa esistenza è connessa direttamente con l'epoca pre- o proto-antropocentrica dello Stato della dominazione politica.

\section{La democrazia e i governi non democratici}

Queste poche note riguardo al governo democratico demarcano il suo ambiente istituzionale e valoriale in opposizione agli altri governi antropocentrici. Mi riferisco nello specifico alla rappresentanza e del tutto secondariamente al governo prerappresentativo.

La democrazia si distingue chiaramente dalla rappresentanza. Hanno come punto d'incontro il fatto che entrambe presuppongono la formazione della società dei cittadini come istituzione organica del governo, cioè come popolo. Si differenziano però per il fatto che la democrazia attribuisce alla società la responsabilità politica totale, mentre la rappresentanza solo lo status di responsabile. Nella democrazia le istituzioni rappresentative si costituiscono e funzionano dal punto di vista del soggetto servitore 
del governo, al contrario che nel governo rappresentativo, che è caratterizzato dalla spartizione della responsabilità politica tra responsabile e rappresentante. La differenza non è soltanto morfologica, ma include gli elementi di un contrasto tipologico significativo.

Per quanto riguarda il sistema politico pre-rappresentativo, nella fattispecie quello della modernità, verifichiamo che non include nessuno degli elementi della rappresentanza né, evidentemente, della democrazia. La società è intesa come un privato, non costituisce una categoria politica istituzionale (popolo) e neppure detiene la minima responsabilità politica. Lo Stato non incarna semplicemente l'interezza del sistema politico, ma si appropria anche del concetto della collettività sociale (ovvero della volontà collettiva, che si esprime attraverso l'idea di nazione), poiché solo in questo modo si legittima ad esercitare la dominazione politica contro la società dei cittadini, cioè allo stesso tempo gli status di rappresentante e di responsabile.

Da quanto sopra, ne consegue che l'obiettivo della democrazia, la realizzazione della libertà totale e parimenti politica, non può essere completato attraverso gli altri due sistemi politici. Da un lato, la rappresentanza appare come un governo transitorio che collega l'epoca proto-antropocentrica a quella della realizzazione antropocentrica. Dall'altro, la pre-rappresentanza è chiamata a preservare la sola libertà individuale e, in misura minore, con il procedere dell'epoca proto-rappresentativa, alcuni diritti sociali e politici, i quali delimitano estensivamente la sua sostanza. Riguardo a questo, la scienza moderna, perseverando ad includere il proprio governo (il suo sistema socio-economico e quello politico) fra le democrazie e, per di più, a dichiarare che è anche democratico e rappresentativo - nel momento in cui rappresentanza e democrazia costituiscono due governi tipologicamente diversi -, si mostra semplicemente come incompleta epistemologicamente e davvero sensibile alle notazioni ideologiche della scienza.

\section{Il tempo della democrazia}

Abbiamo già dimostrato che la libertà totale e, in particolare in questo contesto, quella politica risalgono all'epoca della maturità antropocentrica dell'uomo sociale. Abbiamo notato inoltre che l'ordine di arrivo dell'uomo sociale alla libertà è predeterminato, secondo il principio per cui la libertà individuale viene prima per definizione e la libertà sociale e quella politica seguono 4 .

4 È evidente che questo ordine dello sviluppo antropocentrico interessa la dinamica della protogenesi evolutiva dell'uomo sociale poiché, quando questo processo giunge a compimento, il 
Nella misura in cui l'ordine dei governi segue, come abbiamo visto, l'ordine di sviluppo dei loro obiettivi, nella fattispecie della libertà, è evidente che il tempo della democrazia coincide con quello della maturità antropocentrica, cioè della libertà totale. Non è possibile, ad esempio, che una società, giunta per la prima volta all'antropocentrismo, si sostanzi secondo la modalità della fase del compimento, proprio come nessun uomo può nascere maturo a sessant'anni e in seguito regredire alla propria infanzia. Questo fatto innegabile, che per quanto riguarda l'individuo è d'immediata comprensione, è oggettivamente difficile che diventi percepibile quando riguarda il fenomeno sociale, in particolare nel contesto del cosmosistema antropocentrico, in quanto il secondo è unico, vive una sola volta come tipologia, non più e più volte.

Di conseguenza, l'incapacità oggettiva della scienza contemporanea di capire la biologia del fenomeno sociale non indica che questa non contribuisca come fenomeno e come componente evolutiva. Non è del presente l'incorrere in questo grande rischio. Riguardo a ciò, comunque, è sufficiente evidenziare alcune delle sue manifestazioni. Il tempo di un governo, e dunque anche della democrazia, non è connesso alle funzioni mentali o cognitive dell'uomo. Non basta comprendere il concetto di uno specifico governo perché questo diventi in seguito oggetto di applicazione. Direi che non è possibile che il concetto venga compreso senza che contribuiscano i suoi presupposti o ci siano le circostanze mentali che possano condurre verso la capitalizzazione dell'esperienza storica. Se ad esempio si tenta l'applicazione della democrazia ai nostri giorni, emergerà un risultato diverso, proprio come nel caso dell'uscita dell'Europa occidentale dal cosmosistema despotico. Lo stesso è avvenuto anche con il trasferimento del fenomeno delle confederazioni/città nell'Europa occidentale durante il Rinascimento, quando i governi associati le adattarono alla loro prematura conclusione antropocentrica. Era l'epoca in cui le enclaves antropocentriche dell'Europa rinascimentale erano convinte che avrebbero rifondato quella che era, a loro avviso, l'insuperabile "Antichità". All'interno di questo tentativo si è generato un fenomeno antropocentrico appropriato alle nuove circostanze: il classicismo nell'architettura, nella musica, nel teatro e via dicendo. Fenomeni analoghi si incontrano nell'economia, nella politica, nella società in generale, ai quali però ha contribuito anche la differenza di fase, che ha contrapposto l'Europa occidentale moderna a quella dell'epoca pre-soloniana proto-antropocentrica del mondo ellenico.

cambiamento di governi obbedisce a cause differenti, come anche nei casi in cui entità sociali marginali di tipo despotico si avvicinano al divenire antropocentrico. 
Si spiega così perché non solo non si incontri ai nostri giorni un governo democratico, ma anche perché la scienza moderna sia incapace di comprenderlo. In altre parole, la posta in gioco ai nostri giorni oscilla tra il governo pre-rappresentativo e la sua deviazione autocratica. La democrazia non vi prende parte, non perché qualcuno la sottragga dalle società, ma poiché non sussiste una sua richiesta: non è neppure inclusa come azzardo nel sistema di valori dell'uomo contemporaneo, né vi concorrono i suoi presupposti fattuali.

Di conseguenza, in accordo con quanto sopra, l'ordine della maturità e, per estensione, dello sviluppo delle forme di governo nel cosmosistema antropocentrico, è il seguente: pre-rappresentativo, rappresentativo, democratico. Quello prerappresentativo tende a conservare la semplice libertà individuale, senza toccare il fondamentale basarsi sulla proprietà del sistema (economico e politico). Lo adegua solo alle peculiarità della necessità proto-antropocentrica, limitando quindi la proprietà agli oggetti (attraverso la produzione e via dicendo), non estendendola dunque all'uomo sociale. Riguardo a ciò, la privazione della libertà sociale e politica è chiamata ad essere verificata sulla base del contratto, cioè del consenso e letteralmente della chiara rinuncia a questa, non necessaria. La fase proto-antropocentrica pone i fondamenti basilari della nuova epoca, assieme al concetto che su questa si edificano i parametri del cosmosistema familiare.

In ogni caso, il governo pre-rappresentativo è chiaramente non rappresentativo. Il nome che lo definisce rivela solo che si inserisce in una dinamica che a lungo andare condurrà al governo rappresentativo. Tanto più evolvono i parametri che muovono lo sviluppo antropocentrico, quanto più prenderà forma la pretesa dell'uomo sociale di svincolarsi dalle servitù/dipendenze che sono conformi con la proprietà sul sistema 0 anche di entrare in questo, al fine di controllarlo e di liberarsi dalla dipendenza. Il governo rappresentativo è situato al confine tra l'epoca proto-antropocentrica e quella pienamente antropocentrica. L'uomo sociale prende parte alla libertà sociale e politica, ma in misura ristretta, finché non raggiunge la fase finale della realizzazione antropocentrica, la libertà totale e, di conseguenza, la democrazia 5 .

Non è necessario ai fini di questo approccio occuparsi della base eziologica della democrazia. È sufficiente notare che l'ingresso graduale della società dei cittadini nel governo corrisponde proporzionalmente al rendersi autonomo del parametro economico dal dominio sociale e dal suo sviluppo al livello del cosmosistema. Di

\footnotetext{
$5 \mathrm{Nel}$ presente studio l'epistemologia della democrazia viene esaminata esclusivamente nel contesto della fase cratocentrica. Viene pertanto lasciata da parte la fase dell'ecumene postcratocentrica e, di conseguenza, la democrazia nel contesto della cosmopoli/Stato-mondo.
} 
conseguenza, l'iniziale pretesa costituzionale della società dei cittadini nasce come frutto della necessità di controbilanciare la crescente forza politica della proprietà economica, necessità che con il tempo si trasforma in materia di libertà, in progetto stesso di vita. Considerazioni analoghe valgono anche per la libertà sociale, anche se il suo percorso dipenderà dalle manifestazioni specifiche della relazione che si crea tra il settore del lavoro e il settore del sistema dell'economia. Entrambe però, la libertà sociale e quella politica, la stessa realtà antropocentrica nel suo insieme, obbediscono al parametro del sistema comunicativo. Nel mondo antropocentrico noto il sistema comunicativo dipende radicalmente dalla scala cosmosistemica. Quella piccola per quanto riguarda in particolare il cosmosistema ellenico/antropocentrico, quella grande per quanto riguarda la grande scala cosmosistemica dello Stato-nazione. La focalizzazione sulla scala mostra proprio come questa sia significativa dal momento che costituisce la premessa, tuttavia non esclusiva, affinché il fatto sociale si inserisca nel divenire antropocentrico. Senza entrare nel dettaglio nella verifica della considerazione, richiamerò il modello ellenico. La scala è rimasta stabilmente la stessa dagli anni della Creta micenea fino all'età classica. La democrazia però è entrata nell'orbita antropocentrica della città solo nel V secolo, cioè nel momento in cui sono maturati i parametri che fondano e muovono il divenire antropocentrico nella storia ${ }^{6}$. Per la stessa ragione, l'assenza di democrazia quanto di rappresentanza nella nostra epoca non è dovuta alla grande scala cosmosistemica, ma allo stadio proto-antropocentrico che l'uomo sociale attraversa in questa.

\section{L'epoca della modernità e la prospettiva della democrazia}

Dalle poche cose accennate sopra risulta evidente come la nostra epoca stia vivendo la fase proto-antropocentrica del cosmosistema antropocentrico e, quindi, le circostanze del governo pre-rappresentativo. L'asserzione della scienza moderna secondo cui il mondo della nostra epoca sarebbe transitato direttamente dal periodo despotico a quello della maturità antropocentrica, cioè della democrazia, viene dunque smontata in quanto insostenibile. Da questo punto di vista, l'ostinazione della scienza moderna nell'includere un governo proto-antropocentrico modello, e dunque il suo

\footnotetext{
${ }^{6}$ Anche i pre-Greci, come pure alcuni popoli asiatici, hanno dato vita a città; lo sviluppo antropocentrico e, inoltre, la democrazia si inseriscono però come fenomeno nell'orbita del mondo greco, cioè nella misura in cui le comunità delle città si sono inserite in una chiara orbita cosmosistemica. La tematica della scala è stata trattata esaustivamente da Aristotele. Come emerge, però, i Greci avevano piena consapevolezza del suo significato per la sostanziazione antropocentrica e per l'evoluzione dell'uomo sociale.
} 
sistema politico, di base un'oligarchia elettiva $\mathrm{o}$, più precisamente, governata da un solo individuo, fra le democrazie, si iscrive in principio nella sua più generale incapacità nel distinguere fra la scala cosmosistemica, la quale è evidentemente superiore e per questo creatrice di parametri (di quello comunicativo e di quello economico, tra gli altri) che superano di gran lunga quelli di piccola scala, e lo stadio antropocentrico che attraversa. Così si spiega come il concetto di modernità, che doveva definire l'epoca antropocentrica moderna, finisca per rivelarsi una chiara scelta ideologica che pone il mondo moderno nella scala della realizzazione antropocentrica e, inoltre, come misura di valutazione del passato e al "termine" evolutivo del divenire antropocentrico. Proprio questa combinazione di rappresentazione ideologica della nostra epoca ed evidente carenza epistemologica spiega anche perché sia del tutto assente dalla scienza contemporanea la prospettiva dello sviluppo, non solo come fase del generale divenire antropocentrico, ma anche come problematica per la risoluzione dei problemi che sopravvengono ai nostri giorni. Ciò insegna dunque che le soluzioni ai problemi che sopraggiungono devono essere cercate "causalmente" all'interno del sistema corrente e non al di fuori di questo.

Tale fatto diventa evidente qualora qualcuno consideri l'ostinazione della modernità nell'ignorare il carattere degli sviluppi che sono emersi in misura ridotta e hanno reso manifesta la propria presenza a partire dagli anni Ottanta, con l'evoluzione globale dei parametri fondamentali (dell'economia, della comunicazione e via dicendo) del cosmosistema antropocentrico e, di conseguenza, il rovesciamento radicale dell'equilibrio tra la società, l'economia e la politica.

La domanda seguente torna alla fonte della nostra problematica. Occorre, mi chiedo, convenire che la transizione verso la grande scala cosmosistemica segni negativamente la prospettiva della transizione verso la democrazia? La risposta, stando alla scienza moderna, è "sì", a maggior ragione poiché, ai fini della propria dimostrazione, adduce non solo l'argomentazione della scala e della complessità ma anche quella del carattere pre-moderno della democrazia "ellenica". Dal momento che ha la meglio l'affermazione secondo cui la libertà (individuale) dei «moderni» sia superiore alla libertà (totale) degli «antichi» e di conseguenza che l'oligarchia governata da un solo individuo (la cosiddetta democrazia rappresentativa) della nostra epoca sia superiore alla semplice democrazia, è superfluo qualunque discorso riguardante la transizione verso la democrazia, dato che tale discorso si eguaglierà alla regressione e in ogni circostanza è riconducibile alla semiologia del populismo!

$\mathrm{Al}$ contrario, l'epistemologia cosmosistemica insegna che la nostra epoca è entrata in un'orbita antropocentrica ordinata in modo cosmosistemico che pregiudica essa 
stessa la sua semiologia evolutiva. La differenza di scala in relazione alla sua matrice antropocentrica, il cosmosistema ellenico, non cancella la sua posta in gioco. Indica solo che nel cammino verso il compimento verrà chiamata a perseguire differenti soluzioni, nell'ambito cioè di una realizzazione teleologica e, nello specifico, mediante la tecnologia e non l'incontro fisico fra gli uomini.

Da questo punto di vista, il fatto che la nostra epoca venga classificata nella fase proto-antropocentrica del divenire sociale spiega chiaramente il carattere prerappresentativo del tipo di governo, così come la formazione del sistema economico non basata sul principio della libertà sociale, cioè della relazione fra il settore del lavoro e questo sistema economico. Tale realtà, ma in particolare gli sviluppi che vengono registrati a partire dagli anni Ottanta, prefigurano l'inizio di un nuovo periodo, riguardo al quale i parametri fondamentali antropocentrici (l'economia, la comunicazione e via dicendo) sono già transitati nel futuro, con il proprio sviluppo oltre lo Stato, al livello del cosmosistema totale, d'ora in avanti globale. Il fatto che il sistema (economico, sociale e politico) continui a rimanere prigioniero del passato dell'epoca dell'Illuminismo non confuta la dinamica del fenomeno. Conferma semplicemente che la sequenza del cambiamento del contesto antropocentrico nel percorso verso la sua realizzazione non è lineare. Avviene secondo le relazioni, le quali favoriscono dapprima coloro che sono più prossimi alla produzione dei grandi eventi e che hanno familiarità con questi. Questo avviene ai nostri giorni, dal momento che le società intendono muoversi nel tempo storico secondo i valori, le ideologie, i comportamenti e le istituzioni dell'epoca della transizione dal despotismo all'antropocentrismo, le quali si rifiutano di mettere in discussione le loro certezze e di cercare modi per restaurare l'equilibrio, ovvero la loro influenza sugli avvenimenti politici. Nello specifico, trovano il proprio coinvolgimento nei processi politici nel diritto ad intervenire in modo extraistituzionale nella politica (per esempio attraverso proteste) e non in qualità di associati del governo, ovvero di soci nel processo dell'attività decisionale politica. Certamente l'epistemologia cosmosistemica espone una conclusione che spicca per il suo significato: che più l'economia muta e diviene autonoma dallo stretto contesto della città, che si sviluppa nel complesso del cosmosistema, ossia a livello internazionale, più le società di cittadini cercano di ottenere il proprio ingresso nel governo, al fine di controbilanciare la sua forza e di focalizzare l'obiettivo della politica sui loro interessi. Il che suggerisce come l'allargamento del campo della libertà dal suo contesto individuale a quello socio-economico e politico sia connesso a sua volta con lo sviluppo di quelle 
relazioni che optano per «il potere massimo del governo» 7 . Questo ragionamento rivela chiaramente come la crisi che si verifica ai nostri giorni non riguardi la realizzazione della transizione proto-antropocentrica che ha avuto avvio dall'uscita dal Medioevo, ma il suo superamento. La nuova fase in cui sta entrando il mondo oggi si caratterizzerà per la pretesa delle società di trasformarsi in socie del governo, che si concretizza nella trasformazione dell'oligarchia governata da un solo individuo in un governo rappresentativo e, a lungo termine, in una democrazia. La prospettiva del superamento della fase cratocentrica che la nostra epoca attraversa e della transizione verso l'ecumene (post-cratocentrica) e verso la cosmopoli (Stato-mondo) relativa a questa, si situa ancor più lontano nell'orizzionte della cosmostoria ${ }^{8}$.

Possiamo attingere la testimonianza cognitiva di questo sviluppo dal modello antropocentrico ellenico già compiutosi. L’analogia nella comparazione, alla quale ci chiama la differenza di scala cosmosistemica, ci guida infine alla verifica che il progressivo sviluppo dei parametri antropocentrici conduce le società a rivendicare il proprio ingresso nel governo al fine di compensare la propria incapacità socioeconomica e politica. Allo stesso tempo emerge però la base dell'analogia. Ad esempio, la comparazione fra il parametro economico della grande scala cosmosistemica e quello della piccola deve avvenire non in termini quantitativi, come fa la scienza moderna (per esempio distinguendo tra economia "industriale" e "artigianale"), ma alla luce del risultato che questa produce nell'ambiente sociale familiare. Verificheremo allora che l'economia della nostra epoca produce un risultato socio-economico e politico equivalente a quello dell'epoca di Solone. Non tocca, però, né il governo rappresentativo soloniano né, chiaramente, la democrazia. Per quanto riguarda il parametro comunicativo, considerazioni simili potrebbero essere riferite da chiunque.

Da quanto sopra, risulta che espressioni come democrazia diretta ed indiretta, democrazia rappresentativa, democrazia partecipativa e altre, contengono chiaramente i desiderata ideologici e la carenza epistemologica della modernità, ma non restituiscono da sole la natura fattuale della forma di governo della nostra epoca. Lo stesso vale anche per la chiara reticenza sul fatto che la piega sociale e politica della libertà si definisca dal punto di vista del diritto eteronomo e non dell'autonomia.

Da questo punto di vista, la necessità che la scienza moderna si liberi dalle rigidità ideologiche dell'epoca della transizione e, nella fattispecie, dell'Illuminismo, per potersi

\footnotetext{
7 ARISTOTELE, Politica, IV 1289a.

8 Approfondimenti sulla tipologia cosmosistemica dello sviluppo in altre mie opere quali To

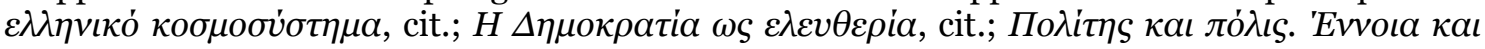

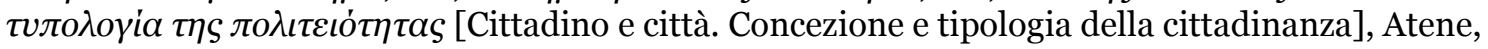
Edizioni Papazisis, 2003.
} 
liberare dalle proprie certezze e poter entrare nello stadio della costituzione di una nuova epistemologia con pretesa cosmosistemica, si dimostra urgente. Tale posta in gioco riguarda tanto i concetti, la tipologia e il tempo del fatto sociale quanto il ripensamento della cosmostoria. D'altronde, riteniamo che questo ripensamento della cosmostoria dentro alla visione cosmosistemica dell'uomo sociale condurrà al recupero del passato come modello, così come che la sua valutazione porterà alla scienza inestimabili informazioni nel tentativo di costituzione di una nuova epistemologia globale, cioè cosmosistemica per quanto riguarda la posta in gioco. 


\section{* L'autore}

Georgios Contogeorgis, professore dell'università Panteion di Atene, è stato rettore nello stesso ateneo, direttore di ricerca del Centro Nazionale per la ricerca scientifica francese (CNRS), e per molti anni visiting professor presso l'Istituto di Studi Politici (IEP) di Parigi, titolare della cattedra Francqui presso l'Université Libre de Bruxelles, membro del Consiglio superiore e del Consiglio per la ricerca dell'Istituto universitario europeo (EUI) di Firenze. Esperto in materia universitaria nell'Unione europea è stato uno dei membri fondatori di EPSNET e della Società di scienze politiche greca (IPSA). Ha insegnato come visiting professor presso numerose università straniere, mentre i lavori sono stati pubblicati in molti paesi. È membro del Consiglio Scientifico e docente del Master di Studi Europei dell'Università di Siena, dottore honoris causa dell'Università di Oradea e membro corrispondente dell'Accademia Internazionale di Cultura

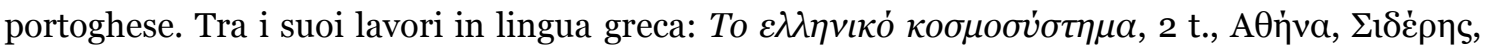

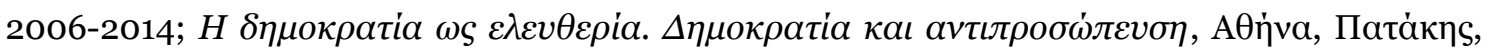

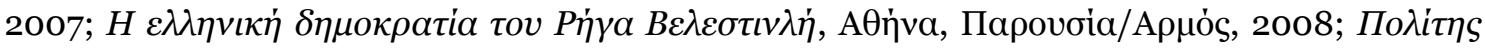

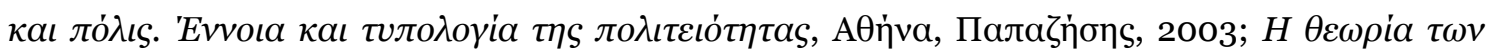

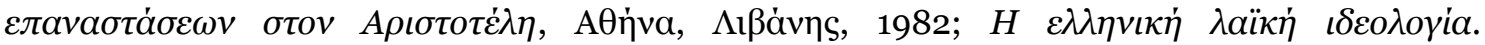

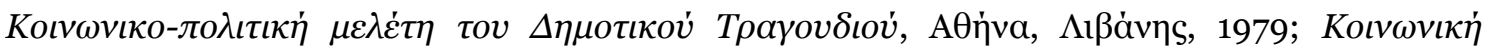

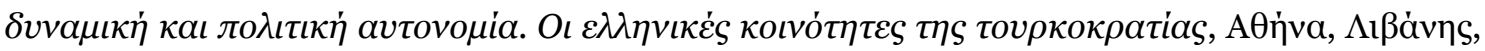

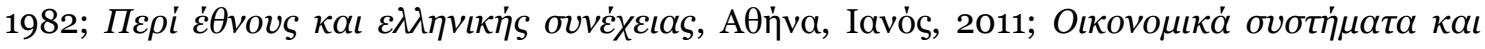

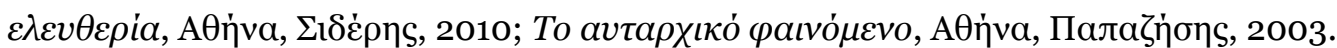

URL: < http://www.studistorici.com/progett/autori/\#Contogeorgis >

\section{Per citare questo articolo:}

CONTOGEORGIS, Georgios, «Epistemologia della democrazia e modernità. La posta in gioco del superamento dell'llluminismo in Europa occidentale», Diacronie. Studi di Storia Contemporanea : Stato, costituzione e democrazia, 29/09/2016,

URL:< http://www.studistorici.com/2016/09/29/contogeorgis_numero_27/ >

Diacronie Studi di Storia Contemporanea $\vartheta$ www.diacronie.it

Risorsa digitale indipendente a carattere storiografico. Uscita trimestrale. redazione.diacronie@hotmail.it

Comitato di redazione: Jacopo Bassi - Luca Bufarale - Elisa Grandi - Antonio César Moreno Cantano - Deborah Paci - Fausto Pietrancosta - Alessandro Salvador - Matteo Tomasoni - Luca Zuccolo

Diritti: gli articoli di Diacronie. Studi di Storia Contemporanea sono pubblicati sotto licenza Creative Commons 3.0. Possono essere riprodotti e modificati a patto di indicare eventuali modifiche dei contenuti, di riconoscere la paternità dell'opera e di condividerla allo stesso modo. La citazione di estratti è comunque sempre autorizzata, nei limiti previsti dalla legge. 